\title{
Incidence and risk of eribulin mesylate related hematologic toxicity
}

\author{
Bin Zhao ${ }^{1}$ and Hong Zhao ${ }^{2}$ \\ 1The Second Affiliated Hospital and Yuying Children's Hospital, Wenzhou Medical University, Wenzhou 325027, Zhejiang, \\ China \\ ${ }^{2}$ Department of Medical Oncology, The Third Affiliated Hospital of Harbin Medical University, Harbin 150081, Heilongjiang, \\ China
}

Correspondence to: Bin Zhao, email: doctorbinzhao@126.com

Hong Zhao, email: doctorhongzhao@126.com

Keywords: eribulin mesylate, hematologic toxicity, cancer, adverse event

Received: August 16, $2017 \quad$ Accepted: October 05, $2017 \quad$ Published: December 15, 2017

Copyright: Zhao et al. This is an open-access article distributed under the terms of the Creative Commons Attribution License 3.0 (CC BY 3.0), which permits unrestricted use, distribution, and reproduction in any medium, provided the original author and source are credited.

\section{ABSTRACT}

Eribulin mesylate, a microtubule dynamics inhibitor, has been approved for the treatment of several malignancies. Due to its novel mechanism of action, eribulin is often associated with a distinct profile of adverse events including hematologic toxicities. Here we searched PubMed and Embase database from reception to August 2017 for clinical trials with eribulin treatment. Eligible studies included trials with eribulin administered at a standard dose of $1.4 \mathrm{mg} / \mathrm{m}^{2}$ intravenously on days 1 and 8 in a 21-day cycle, and adequate safety data profile reporting neutropenia, leucopenia, anemia, febrile neutropenia and thrombocytopenia. The overall incidence, relative risk (RR) and $95 \%$ confidence interval (CI) were calculated. A total of 39 studies with 6,092 subjects were included in this study. The incidences of eribulin related all-grade and high-grade hematologic toxicities were: neutropenia, 56\% and $39 \%$; leucopenia, $44 \%$ and $21 \%$; anemia, $33 \%$ and $2 \%$; febrile neutropenia, $5 \%$ and $5 \%$; and thrombocytopenia, $12 \%$ and $12 \%$. Compared with controls, eribulin was associated with a significant increased risk of all-grade ( $R R, 1.94 ; 95 \%$ CI, 1.37-2.74) and high-grade ( $R R, 2.58 ; 95 \% C I, 1.30-5.10)$ neutropenia, all-grade ( $R R, 1.81 ; 95 \%$ CI, 1.10-2.97) and high-grade (RR, 2.95; 95\% CI, 1.36-6.39) leucopenia, but not with anemia and febrile neutropenia. Trial sequential analysis showed the results from neutropenia, leucopenia and anemia established sufficient and conclusive evidence. Our study suggested that eribulin therapy, compared with control, was associated with an increased risk of hematologic toxicities. Hence hematologic monitoring at regular intervals should be advised.

\section{INTRODUCTION}

Eribulin mesylate (hereafter referred as "eribulin"), a macrocyclic ketone analogue of Halichondrin $\mathrm{B}$, is a completely synthetic, microtubule dynamics inhibitor $[1,2]$. Unlike traditional tubulin-targeting agents which usually suppress growth and depolymerization of microtubules, eribulin inhibits microtubule polymerization through a special binding site on B-tubulin [3]. Preclinical evidence further reveals that eribulin suppresses the development of tumor by inhibiting mitotic spindle formation [3], reversing phenotype from epithelial-mesenchymal transition state to mesenchymal-epithelial transition state [4], and remodeling tumor vasculature [5]. Currently, eribulin has been approved by the US Food and Drug Administration (FDA) for the treatment of breast cancer (BC) [6], and recently, liposarcoma [7]. Additionally, it is reported that eribulin is being investigated in several malignancies such as non-small cell lung cancer, pancreatic cancer, head and neck cancer, prostate cancer, and ovarian cancer [8]. Accordingly the increase in the application of eribulin is expected in the future. 
Due to its novel mechanism, eribulin treatment is often associated with a distinct profile of adverse events. Previous studies have showed that the common side effects of eribulin were myelosuppression, fatigue/ asthenia, alopecia, and peripheral neuropathy [9-14]. Although hematologic toxicities associated with eribulin have been reported, there has been no systematic attempt to synthesize these data and the relative risk of hematologic toxicities induced by eribulin has yet to be evaluated. Therefore, here we conducted a metaanalysis of available clinical data to calculate the overall incidence and relative risk of developing hematologic toxicities, namely neutropenia, leucopenia, anemia, febrile neutropenia, and thrombocytopenia, in cancer patients treated with eribulin.

\section{RESULTS}

\section{Search results}

A total of 796 potentially relevant articles were found during the initial search, including 395 studies from PubMed and 401 trials from Embase. 361 articles were excluded because of duplications. After careful screening of titles and abstracts, 369 studies were removed since they did not meet the eligible criteria. Further reviewing the whole texts of the remaining 66 potentially eligible articles, 27 were not included because of different dose of eribulin $(n=19)$, insufficient data $(n=5)$ and duplication $(n=3)$. A total of 39 studies were enrolled for the final analysis. 33 were single arm trials [15-47], the other 6 were RCTs [9-14]. A flow chart was presented in Figure 1.

\section{Population characteristics}

6,092 patients were included in this meta-analysis (eribulin, 4,958; control, 1,134). 5,098 patients had breast cancer (eribulin, 4,227; control, 871) from 31 studies. 630 patients had soft tissue sarcoma (STS, eribulin, 406; control, 224) from 3 studies. 146 patients had non-small cell lung cancer (NSCLC, eribulin, 107; control, 39) from 2 studies. 105 patients had prostate cancer (PC; eribulin, 105; control, 0) from 1 study. 73 patients had ovarian cancer (OC; eribulin, 73; control, 0) from 1 study. 40 patients had head and neck cancer (HNC; eribulin, 40; control, 0) from 1 study. The dose and schedule of eribulin was $1.4 \mathrm{mg} / \mathrm{m}^{2}$ in 2-5 minutes intravenously on days 1 and 8 on a 21-day schedule, the currently FDA-recommended dose until unacceptable toxicity, disease progression or patient refusal. In one study [14], pharmacokinetic and pharmacodynamical analysis revealed that $0.9 \mathrm{mg} /$ $\mathrm{m}^{2}$ is the optimal dose for NSCLC patients. The median treatment ranged from 1.5 months [34] to 7.7 months [23]. The basic clinicopathological characteristics of eligible trials were summarized in Table 1. The numbers of hematologic toxicities were presented in Table 2. It should be noted that not all studies consistently illustrated the five adverse event (AE) of our interest.

\section{Overall incidence of hematological toxicity}

A total of 4,958 patients from 39 non-randomized trials and treatment arms in RCTs were included. The overall incidences of all-grade neutropenia, leucopenia, anemia, febrile neutropenia and thrombocytopenia in patients treated with eribulin were 56\% (95\% CI, 46\%-65\%), 44\% (95\% CI, 30\%-59\%), 33\% (95\% CI, 25\%-40\%), 5\% (95\% CI, 3\%-7\%) and 12\% (95\% CI, $8 \%-15 \%$ ), respectively (Table 3 ). Whereas the summary incidences of high-grade (grade III and grade IV) neutropenia, leucopenia, anemia, febrile neutropenia and thrombocytopenia were $39 \% \quad(30 \%-48 \%), 21 \%$ (95\% CI, 14\%-28\%), 2\% (95\% CI, 1\%-3\%), 5\% (95\% CI, 3\%-6\%), and 12\% (95\% CI, 6\%-18\%), respectively (Table 4). Significant heterogeneities were observed in the calculation of these hematological AEs. Therefore, the random-effects models were applied. Since most of these eligible trials were conducted in patients with breast cancer, the hematological toxicities of eribulin stratified by different cancer type were examined (Table 3 and Table 4). The incidences of all-grade $(p=0.76)$ and highgrade $(p=0.54)$ neutropenia were similar in BC patients and non-BC patients. Similarly, the incidences of all-grade $(p=0.69)$ and high-grade $(p=0.38)$ leucopenia did not show statistical difference between BC patients and non$\mathrm{BC}$ patients. Meanwhile, the incidences of both all-grade $(p=0.004)$ and high-grade $(p=0.04)$ anemia in non$\mathrm{BC}$ patients were statistically higher compared with $\mathrm{BC}$ patients. In contrast, the incidences of all-grade $(p=0.01)$ and high-grade $(p=0.02)$ thrombocytopenia in non-BC patients were lower compared with BC patients.

\section{Relative risk of hematologic adverse events}

The RRs and their 95\% CIs of hematologic toxicities were calculated with 6 RCTs ( 3 phase III studies and 3 phase II studies including 2,546 patients) [9-14]. The relative risks of all-grade neutropenia, leucopenia, anemia, febrile neutropenia and thrombocytopenia were 1.94 (95\% CI, 1.37-2.74; $p=0.01), 1.81$ (95\% CI, 1.10-2.97; $p=0.02), 0.95$ (95\% CI, 0.82-1.11, $p=0.53), 2.15(95 \%$ CI, 0.79-5.82; $p=0.12$ ), and 0.21 (95\% CI, 0.12-0.36; $p<0.001$ ) respectively (Figure 2 ). The relative risk of high-grade neutropenia, leucopenia, anemia, febrile neutropenia and thrombocytopenia in patients treated with eribulin were $2.58(95 \% \mathrm{CI}, 1.30-5.10 ; p=0.02), 2.95$ (95\% CI, 1.36-6.39; $p=0.01), 0.77$ (95\% CI, 0.52-1.14; $p=0.19), 2.15$ (95\% CI, 0.79-5.82; $p=0.12)$, and 0.03 (95\% CI, $0.00-0.21 ; p<0.001)$ respectively (Figure 3 ). The fixed-effects models were applied in the analysis of both all-grade and high-grade anemia, febrile neutropenia, and thrombocytopenia because there was no evidence of 
heterogeneity for these outcomes. Additional analyses using the random-effects models yielded similar results. Substantial heterogeneities were observed in RR calculation of all-grade and high-grade neutropenia and leucopenia. Sensitivity analyses were then carried out to identify potential sources of heterogeneities and to examine the impact of different exclusion criteria on the overall relative risk. Exclusion of one study [11] in which eribulin was compared with capecitabine did not changed the trend of overall risk estimate, but no significant heterogeneities were observed among the remaining trials (as shown in Table 5).

Trial sequential analysis (TSA) showed that the cumulative $z$ curve crossed both the conventional boundary and the trial sequential monitoring boundary in neutropenia and leucopenia analysis, crossed the futility boundary and entered the futility area in anemia analysis (Figure 4). Both these cases established sufficient and conclusive evidence. Accordingly, further trials were not request and were unlikely change our conclusion. Because of the limited available data for febrile neutropenia and thrombocytopenia analysis, TSA was not conducted.

\section{Publication bias}

Egger's test and Begg's funnel plot were conducted to evaluate the publication bias for RR of both all-grade and high-grade neutropenia, leucopenia, anemia, febrile neutropenia and thrombocytopenia. The shapes of the funnel plots did not show any evidence of obvious asymmetry in all analysis.

\section{DISCUSSION}

To our knowledge, this is the first meta-analysis focused on hematologic toxicities associated with eribulin mesylate. Our data revealed that the overall incidences of eribulin related all-grade and high-grade hematologic toxicities were: neutropenia, 56\% and 39\%; leucopenia, $44 \%$ and $21 \%$; anemia, $33 \%$ and $2 \%$, febrile neutropenia, $5 \%$ and $5 \%$; and thrombocytopenia, $12 \%$ and $12 \%$. In addition, our analysis on RCTs revealed that the relative risk of all-grade neutropenia and leucopenia were approximately two-fold (three-fold for high-grade) in patients with eribulin treatment compared with those in the control arms. In contrast, no significantly increased risks were identified in terms of anemia and febrile neutropenia.

Accumulating evidence reveals that eribulin has many characteristics that makes it distinct from other microtubule-targeting agents [48]. Although still not completely clear, the specific biological effects of eribulin might underlie its unique impact on hematologic toxicities. Preclinical studies showed that

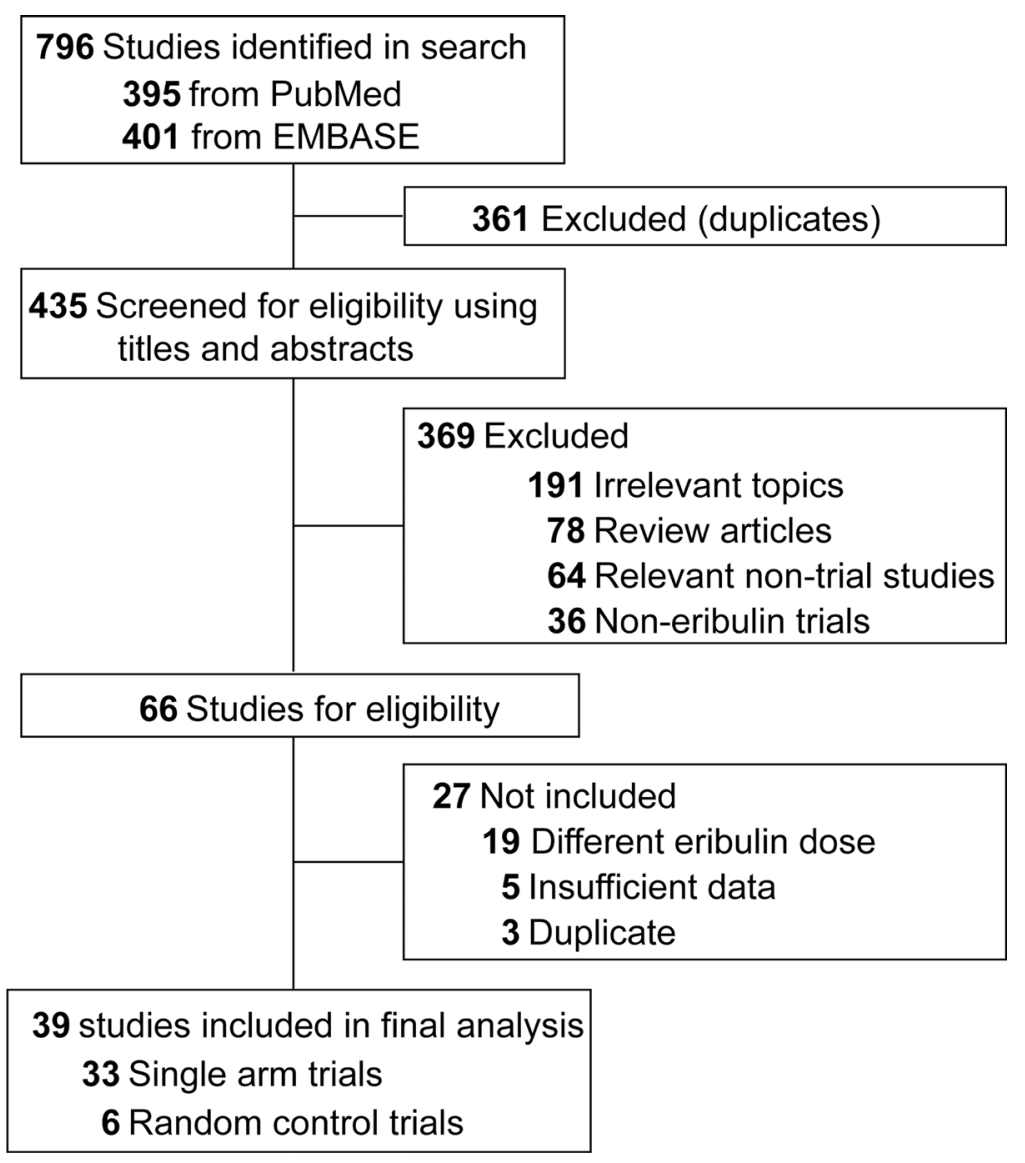

Figure 1: Flow-chart diagram of selected trials included in this meta-analysis. 
Table 1: Baseline clinicopathological characteristics of the clinical trials included in this study

\begin{tabular}{|c|c|c|c|c|c|c|c|c|c|c|c|c|}
\hline Author & Region & Year & $\begin{array}{l}\text { Underlying } \\
\text { malignancy }\end{array}$ & Phase & $\begin{array}{l}\text { Median age } \\
\text { (range), year }\end{array}$ & $\begin{array}{c}\text { Gender } \\
\text { (male/ } \\
\text { female) }\end{array}$ & $\begin{array}{c}\text { ECOG PS } \\
(0 / 1 / 2+)\end{array}$ & $\begin{array}{c}\text { Treatment } \\
\text { duration, } \\
\text { median (range), } \\
\text { month }\end{array}$ & $\begin{array}{l}\text { Median OS }(95 \% \\
\text { CI), month }\end{array}$ & $\begin{array}{c}\text { Median PFS } \\
\text { (95\% CI), } \\
\text { month }\end{array}$ & $\begin{array}{l}\text { Founding } \\
\text { Source }\end{array}$ & $\begin{array}{c}\text { Quality } \\
\text { assessment } \\
\text { (NOS) }\end{array}$ \\
\hline Cortes [9] & Globe & 2011 & $\mathrm{BC}$ & III & $\begin{array}{l}55(28-85) \\
56(27-81)\end{array}$ & $\begin{array}{l}0 / 508 \\
0 / 254\end{array}$ & $\begin{array}{l}217 / 244 / 39 \\
103 / 126 / 22\end{array}$ & $\begin{array}{l}3.9(0.7-16.3) \\
2.1(0.0-21.2)\end{array}$ & $\begin{array}{c}13.1(11.8-14.3) \\
10.6(9.3-12.5)\end{array}$ & $\begin{array}{l}3.7(3.3-3.9) \\
2.2(2.1-3.4)\end{array}$ & Industry & 9 \\
\hline Abraham [10] & US & 2015 & $\mathrm{BC}$ & II & $\begin{array}{l}50(28-70) \\
48(34-67)\end{array}$ & $\begin{array}{l}0 / 30 \\
0 / 19\end{array}$ & $\begin{array}{l}\text { NR } \\
\text { NR }\end{array}$ & $\begin{array}{l}\mathrm{NR} \\
\mathrm{NR}\end{array}$ & $\begin{array}{l}\text { NR } \\
\text { NR }\end{array}$ & $\begin{array}{l}\text { NR } \\
\text { NR }\end{array}$ & Industry & 7 \\
\hline Kaufman [11] & Globe & 2015 & $\mathrm{BC}$ & III & $\begin{array}{l}54(24-80) \\
53(26-80)\end{array}$ & $\begin{array}{l}0 / 554 \\
0 / 548\end{array}$ & $\begin{array}{l}250 / 293 / 11 \\
230 / 301 / 17\end{array}$ & $\begin{array}{l}4.1(0.7-45.1) \\
3.9(0.7-47.4)\end{array}$ & $\begin{array}{l}15.9(15.2-17.6) \\
14.5(13.1-16.0)\end{array}$ & $\begin{array}{l}4.1(3.5-4.3) \\
4.2(3.9-4.8)\end{array}$ & Industry & 9 \\
\hline Vahdat [12] & US & 2013 & $\mathrm{BC}$ & II & $\begin{array}{l}52 \\
57\end{array}$ & $\begin{array}{l}0 / 51 \\
0 / 50\end{array}$ & $\begin{array}{l}21 / 29 / 1 \\
20 / 28 / 2\end{array}$ & $\begin{array}{l}3.8(0.8-23.0) \\
2.6(0.8-12.3)\end{array}$ & $\begin{array}{l}\text { NR } \\
\text { NR }\end{array}$ & $\begin{array}{l}3.5(2.7-4.3) \\
3.2(2.4-6.2)\end{array}$ & Industry & 6 \\
\hline Schoffski [13] & Globe & 2016 & STS & III & $\begin{array}{l}56(28-83) \\
56(24-83)\end{array}$ & $\begin{array}{l}67 / 161 \\
82 / 142\end{array}$ & $\begin{array}{l}111 / 114 / 3 \\
90 / 121 / 13\end{array}$ & $\begin{array}{l}\text { NR } \\
\text { NR }\end{array}$ & $\begin{array}{c}13.5(10.9-15.6) \\
11.5(9.6-13.0)\end{array}$ & $\begin{array}{l}2.6(1.9-2.8) \\
2.6(1.8-2.7)\end{array}$ & Industry & 9 \\
\hline Waller [14]" & Europe & 2015 & NSCLC & II & $\begin{array}{l}59(38-80) \\
60(46-77)\end{array}$ & $\begin{array}{l}25 / 16 \\
26 / 13\end{array}$ & $\begin{array}{c}10 / 31 / 0 \\
3 / 36 / 0\end{array}$ & $\begin{array}{l}\text { NR } \\
\text { NR }\end{array}$ & $\begin{array}{l}14.8 \\
14.3\end{array}$ & $\begin{array}{l}5.4(3.2-9.9) \\
5.9(4.3-7.5)\end{array}$ & Industry & 7 \\
\hline Watanabe [15] & Japan & 2017 & $\mathrm{BC}$ & II & $59(26-88)$ & $2 / 949$ & $481 / 364 / 106$ & $3.5(0.8-14.8)$ & NR & NR & Industry & 8 \\
\hline De Bono [16] & Globe & 2012 & $\mathrm{PC}$ & II & $71(47-91)$ & $105 / 0$ & $48 / 57 / 3$ & $2.6(0.8-35.2)$ & $18.6(1.0-32.4)$ & $2.1(0.1-32.2)$ & Industry & 7 \\
\hline Aftimos [17] & Belgium & 2016 & $\mathrm{BC}$ & II & $55(34-81)$ & $0 / 141$ & NR & $3.0(0.3-21.8)$ & $11.3(8.7-12.3)$ & $3.2(2.7-4.0)$ & Industry & 7 \\
\hline Aogi [18] & Japan & 2012 & $\mathrm{BC}$ & II & $54(31-72)$ & $0 / 80$ & $58 / 21 / 1$ & $2.8(0.0-14.5)$ & $11.1(1.0-25.9)$ & $3.7(0.3-14.8)$ & Non-profit & 8 \\
\hline Arnold [19] & US & 2011 & $\mathrm{HNC}$ & II & $61(44-87)$ & $29 / 11$ & $19 / 21 / 0$ & NR & $7.0(5.0-10.0)$ & $2.6(0.0-13.1)$ & Industry & 6 \\
\hline Cortes [20] & Globe & 2010 & $\mathrm{BC}$ & II & $56(26-80)$ & $0 / 291$ & $108 / 165 / 17$ & $3.0(0.3-20.3)$ & $10.4(0.6-19.9)$ & $2.6(0.0-13.1)$ & Industry & 8 \\
\hline Park [21] & Korea & 2017 & $\mathrm{BC}$ & IV & $51(25-79)$ & $0 / 101$ & $30 / 69 / 2$ & $2.1(0.0-23.8)$ & NR & NR & Industry & 4 \\
\hline Araki [22] & Japan & 2017 & $\mathrm{BC}$ & II & $58(31-76)$ & $0 / 40$ & $10 / 18 / 2$ & $6.0(0.3-18.8)$ & NR & $10.7(5.0-13.0)$ & Industry & 5 \\
\hline Puhalla [23] & US & 2016 & $\mathrm{BC}$ & II & $59(31-81)$ & $0 / 52$ & NR & 7.7 & NR & $11.9(6.0-19.1)$ & Industry & 6 \\
\hline Maeda [24] & Japan & 2017 & $\mathrm{BC}$ & II & $54(31-75)$ & $0 / 47$ & $34 / 13 / 0$ & 5.8 & 17.4 & $4.9(3.5-7.0)$ & None & 5 \\
\hline Takashima [25] & Japan & 2016 & $\mathrm{BC}$ & II & $64(40-75)$ & $0 / 35$ & $28 / 7 / 0$ & $6.0(0.3-15.8)$ & 35.9 & $5.8(4.8-8.1)$ & Industry & 5 \\
\hline Yardley [26] & US & 2016 & $\mathrm{BC}$ & II & $56(32-84)$ & $0 / 65$ & NR & NR & $11.5(9.0-17.3)$ & $4.1(3.2-5.6)$ & Industry & 7 \\
\hline Garrone [27] & Italy & 2016 & $\mathrm{BC}$ & II & $62(33-80)$ & $0 / 113$ & NR & $3.0(0.3-20.3)$ & $11.6(0.6-33.3)$ & $3.3(0.6-26.7)$ & None & 6 \\
\hline Dell'Ova [28] & France & 2015 & $\mathrm{BC}$ & II & $50(18-80)$ & $0 / 258$ & $73 / 133 / 52$ & $3.8(0.3-14.3)$ & $4.0(3.3-4.3)$ & $11.2(9.3-12.1)$ & None & 5 \\
\hline Smith [29] & US & 2016 & $\mathrm{BC}$ & II & $62(28-80)$ & $0 / 67$ & $60 / 7 / 0$ & NR & NR & NR & Industry & 7 \\
\hline Wilks [30] & US & 2014 & $\mathrm{BC}$ & II & $60(31-81)$ & $0 / 52$ & $37 / 14 / 1$ & $7.5(0.0-28.5)$ & NR & $11.6(9.1-13.9)$ & Industry & 7 \\
\hline Gitlitz [31] & US & 2012 & NSCLC & II & $63(35-83)$ & $31 / 35$ & NR & $3.0(0.3-17.3)$ & $11.6(8.2-13.7)$ & $2.7(1.3-3.9)$ & None & 6 \\
\hline Dranitsaris [32] & US & 2015 & $\mathrm{BC}$ & II & $58(36-86)$ & $0 / 90$ & NR & $2.3(0.0-14.0)$ & NR & NR & Industry & 5 \\
\hline Fabi [33] & Italy & 2015 & $\mathrm{BC}$ & II & $58(45-71)$ & $0 / 78$ & NR & $3.8(0.3-13.5)$ & $10.1(8.1-13.0)$ & $4.8(3.4-6.4)$ & Industry & 6 \\
\hline Hensley [34] & US & 2011 & $\mathrm{OC}$ & II & $60(38-80)$ & $0 / 73$ & NR & $1.5(0.3-7.5)$ & 22 & 3 & Non-profit & 6 \\
\hline Ates [35] & Turkey & 2016 & $\mathrm{BC}$ & II & $50(28-67)$ & $0 / 66$ & $29 / 30 / 7$ & $2.3(0.3-6.0)$ & $8.0(6.0-9.9)$ & $5.0(4.1-5.8)$ & None & 5 \\
\hline Kawai [36] & Japan & 2017 & STS & II & $52(28-73)$ & $23 / 28$ & $27 / 24 / 0$ & $3.0(0.8-36.8)$ & $13.2(9.5-18.3)$ & $4.1(2.6-5.6)$ & Industry & 7 \\
\hline Kaklamani [37] & US & 2015 & $\mathrm{BC}$ & II & $53(35-78)$ & $0 / 30$ & NR & NR & NR & NR & Industry & 6 \\
\hline Kessler [38] & Sweden & 2015 & $\mathrm{BC}$ & II & $56(35-74)$ & $0 / 48$ & $36 / 7 / 5$ & $5.3(0.8-15.8)$ & $8.9(4.5-13.0)$ & $4.7(4.2-6.0)$ & Non-profit & 5 \\
\hline Lorusso [39] & Italy & 2017 & $\mathrm{BC}$ & II & $62(33-85)$ & $0 / 91$ & $32 / 46 / 13$ & $2.9(0.2-24.4)$ & $11.6(8.7-16.7)$ & $3.1(2.8-3.5)$ & Industry & 7 \\
\hline McIntyre [40] & US & 2014 & $\mathrm{BC}$ & II & $57(31-85)$ & $0 / 56$ & $32 / 21 / 3$ & 4.5 & NR & $6.8(4.4-7.6)$ & Industry & 6 \\
\hline Gamucci [41] & Italy & 2014 & $\mathrm{BC}$ & II & $62(30-79)$ & $0 / 133$ & NR & $3.8(0.8-11.3)$ & $14.3(11.7-16.8)$ & $4.4(3.7-5.0)$ & None & 5 \\
\hline Moscetti [42] & Italy & 2017 & $\mathrm{BC}$ & II & $64(31-85)$ & $0 / 50$ & NR & $3.8(0.8-12.8)$ & $9.0(5.0-13.0)$ & $4.0(3.0-5.0)$ & None & 6 \\
\hline Prestifilippo [43] & Italy & 2017 & $\mathrm{BC}$ & II & $55(40-76)$ & $0 / 31$ & $3 / 16 / 12$ & NR & $5.5(1.0-16.0)$ & $2.0(0.0-7.8)$ & Industry & 6 \\
\hline Quaquarini [44] & Italy & 2017 & $\mathrm{BC}$ & II & $57(39-74)$ & $0 / 44$ & $21 / 20 / 3$ & $4.5(0.8-11.3)$ & $11.8(1.0-77.3)$ & $4.5(1.0-16.6)$ & Industry & 5 \\
\hline Inoue [45] & Japan & 2016 & $\mathrm{BC}$ & II & $55(34-74)$ & $0 / 51$ & $25 / 22 / 4$ & $3.0(0.3-31.5)$ & $11.7(9.2-14.2)$ & $3.6(2.6-4.6)$ & Non-profit & 7 \\
\hline Schoffski [46] & Europe & 2011 & STS & II & $57(18-83)$ & $62 / 65$ & NR & $3.0(0.8-32.3)$ & NR & 2.6 & Industry & 8 \\
\hline Vahdat [47] & US & 2009 & $\mathrm{BC}$ & II & $52(32-81)$ & $0 / 33$ & $18 / 15 / 0$ & 3 & $9.0(0.5-27.5)$ & $2.6(0.0-15.1)$ & Industry & 8 \\
\hline
\end{tabular}

Abbreviations: BC, breast cancer; HNC, head and neck cancer; NSCLC, non-small cell lung cancer; OC, ovarian cancer; PC, prostate cancer, STS, soft tissue sarcoma; PFS, progress-free survival; OS, overall survival; ECOG PS, European cooperative oncology group performance status; NOS, Newcastle-Ottawa Scale; NR, not reported., eribulin mesylate at the dose of $0.9 \mathrm{mg} / \mathrm{m}^{2}$ on day 1 of every 21 -day cycle.

eribulin decreased the expression of genes related with vascular endothelial growth factor (VEGF), EphrinEphR, Wnt, and Notch signaling pathways which were known to be critical in vascular functions [48]. In vitro evidence suggested that eribulin could down-regulate the expression of VEGF, CA9, Notch4, Dll4 and Efnb2 [5, 48]. In clinic, significantly decreased concentration of deoxyhemoglobin was observed in patients treated with eribulin [49]. Eribulin therapy could also decreased the plasma concentration of transforming growth factor beta 
Table 2: Number of events reported in every trial included in this study

\begin{tabular}{|c|c|c|c|c|c|c|c|c|c|c|c|c|c|c|}
\hline \multirow[t]{2}{*}{ Author } & \multirow[t]{2}{*}{ Year } & \multirow{2}{*}{$\begin{array}{l}\text { Underlying } \\
\text { malignancy }\end{array}$} & \multirow{2}{*}{$\begin{array}{c}\text { No. of } \\
\text { patients }\end{array}$} & \multicolumn{2}{|c|}{ Thrombocytopenia } & \multicolumn{2}{|c|}{ Anemia } & \multicolumn{2}{|c|}{ Neutropenia } & \multicolumn{2}{|c|}{ Leucopenia } & \multicolumn{2}{|c|}{ Febrile neutropenia } & \multirow[t]{2}{*}{ CTCAE } \\
\hline & & & & $\begin{array}{c}\text { All- } \\
\text { grade }\end{array}$ & $\begin{array}{l}\text { High- } \\
\text { grade }\end{array}$ & $\begin{array}{c}\text { All- } \\
\text { grade }\end{array}$ & $\begin{array}{l}\text { High- } \\
\text { grade }\end{array}$ & $\begin{array}{c}\text { All- } \\
\text { grade }\end{array}$ & $\begin{array}{l}\text { High- } \\
\text { grade }\end{array}$ & $\begin{array}{c}\text { All- } \\
\text { grade }\end{array}$ & $\begin{array}{l}\text { High- } \\
\text { grade }\end{array}$ & $\begin{array}{c}\text { All- } \\
\text { grade }\end{array}$ & $\begin{array}{l}\text { High- } \\
\text { grade }\end{array}$ & \\
\hline Cortes [9] & 2011 & $\mathrm{BC}$ & $\begin{array}{l}508 \\
254\end{array}$ & $\begin{array}{l}\text { NR } \\
\text { NR }\end{array}$ & $\begin{array}{l}\text { NR } \\
\text { NR }\end{array}$ & $\begin{array}{l}94 \\
56\end{array}$ & $\begin{array}{c}10 \\
9\end{array}$ & $\begin{array}{c}260 \\
73\end{array}$ & $\begin{array}{c}227 \\
52\end{array}$ & $\begin{array}{c}116 \\
28\end{array}$ & $\begin{array}{l}70 \\
14\end{array}$ & $\begin{array}{l}\text { NR } \\
\text { NR }\end{array}$ & $\begin{array}{l}\text { NR } \\
\text { NR }\end{array}$ & 3.0 \\
\hline Abraham [10] & 2015 & $\mathrm{BC}$ & $\begin{array}{l}30 \\
19\end{array}$ & $\begin{array}{l}\text { NR } \\
\text { NR }\end{array}$ & $\begin{array}{l}\text { NR } \\
\text { NR }\end{array}$ & $\begin{array}{l}\mathrm{NR} \\
\mathrm{NR}\end{array}$ & $\begin{array}{l}\mathrm{NR} \\
\mathrm{NR}\end{array}$ & $\begin{array}{l}7 \\
2\end{array}$ & $\begin{array}{l}5 \\
0\end{array}$ & $\begin{array}{l}\mathrm{NR} \\
\mathrm{NR}\end{array}$ & $\begin{array}{l}\mathrm{NR} \\
\mathrm{NR}\end{array}$ & $\begin{array}{l}1 \\
0\end{array}$ & $\begin{array}{l}1 \\
0\end{array}$ & 4.0 \\
\hline Kaufman [11] & 2015 & $\mathrm{BC}$ & $\begin{array}{l}554 \\
548\end{array}$ & $\begin{array}{l}\text { NR } \\
\text { NR }\end{array}$ & $\begin{array}{l}\text { NR } \\
\text { NR }\end{array}$ & $\begin{array}{c}104 \\
96\end{array}$ & $\begin{array}{c}11 \\
6\end{array}$ & $\begin{array}{c}295 \\
87\end{array}$ & $\begin{array}{c}249 \\
27\end{array}$ & $\begin{array}{c}171 \\
57\end{array}$ & $\begin{array}{l}82 \\
11\end{array}$ & $\begin{array}{c}11 \\
5\end{array}$ & $\begin{array}{c}11 \\
5\end{array}$ & 3.0 \\
\hline Vahdat [12] & 2013 & $\mathrm{BC}$ & $\begin{array}{l}51 \\
50\end{array}$ & $\begin{array}{l}\mathrm{NR} \\
\mathrm{NR}\end{array}$ & $\begin{array}{l}\mathrm{NR} \\
\mathrm{NR}\end{array}$ & $\begin{array}{l}13 \\
10\end{array}$ & $\begin{array}{l}3 \\
3\end{array}$ & $\begin{array}{l}24 \\
14\end{array}$ & $\begin{array}{l}16 \\
10\end{array}$ & $\begin{array}{l}\mathrm{NR} \\
\mathrm{NR}\end{array}$ & $\begin{array}{l}\mathrm{NR} \\
\mathrm{NR}\end{array}$ & $\begin{array}{l}\text { NR } \\
\text { NR }\end{array}$ & $\begin{array}{l}\mathrm{NR} \\
\mathrm{NR}\end{array}$ & 3.0 \\
\hline Schoffski [13] & 2016 & STS & $\begin{array}{l}228 \\
224\end{array}$ & $\begin{array}{l}13 \\
62\end{array}$ & $\begin{array}{c}1 \\
34\end{array}$ & $\begin{array}{l}67 \\
69\end{array}$ & $\begin{array}{l}16 \\
27\end{array}$ & $\begin{array}{l}99 \\
53\end{array}$ & $\begin{array}{l}80 \\
35\end{array}$ & $\begin{array}{l}36 \\
23\end{array}$ & $\begin{array}{l}23 \\
10\end{array}$ & $\begin{array}{l}\text { NR } \\
\text { NR }\end{array}$ & $\begin{array}{l}\text { NR } \\
\text { NR }\end{array}$ & 4.02 \\
\hline Waller [14] & 2015 & NSCLC & $\begin{array}{l}41 \\
39\end{array}$ & $\begin{array}{l}\text { NR } \\
\text { NR }\end{array}$ & $\begin{array}{l}\mathrm{NR} \\
\mathrm{NR}\end{array}$ & $\begin{array}{c}8 \\
15\end{array}$ & $\begin{array}{l}4 \\
4\end{array}$ & $\begin{array}{l}12 \\
11\end{array}$ & $\begin{array}{l}7 \\
7\end{array}$ & $\begin{array}{l}3 \\
7\end{array}$ & $\begin{array}{l}1 \\
2\end{array}$ & $\begin{array}{l}\text { NR } \\
\text { NR }\end{array}$ & $\begin{array}{l}\mathrm{NR} \\
\mathrm{NR}\end{array}$ & NR \\
\hline Watanabe [15] & 2017 & $\mathrm{BC}$ & 951 & 33 & 17 & 63 & 35 & 633 & 569 & 593 & 480 & 73 & 73 & 3.0 \\
\hline De Bono [16] & 2012 & $\mathrm{PC}$ & 105 & 8 & 2 & 30 & 2 & 45 & 33 & 29 & 13 & 4 & 4 & 3.0 \\
\hline Aftimos [17] & 2016 & $\mathrm{BC}$ & 141 & 6 & 2 & 15 & 1 & 60 & 52 & NR & NR & 13 & 13 & 4.0 \\
\hline Aogi [18] & 2012 & $\mathrm{BC}$ & 80 & NR & NR & $\mathrm{NR}$ & NR & 80 & 77 & 80 & 60 & NR & NR & 3.0 \\
\hline Arnold [19] & 2011 & $\mathrm{HNC}$ & 40 & NR & NR & 20 & 1 & 13 & 4 & 12 & 5 & NR & NR & 3.0 \\
\hline Cortes [20] & 2010 & $\mathrm{BC}$ & 291 & NR & NR & 82 & 6 & 174 & 157 & 64 & 41 & 16 & 16 & 3.0 \\
\hline Park [21] & 2017 & $\mathrm{BC}$ & 101 & NR & NR & 12 & 3 & 92 & 90 & 11 & 6 & NR & NR & 4.03 \\
\hline Araki [22] & 2017 & $\mathrm{BC}$ & 30 & 14 & 6 & 22 & 0 & 27 & 20 & 28 & 6 & NR & NR & 4.0 \\
\hline Puhalla [23] & 2016 & $\mathrm{BC}$ & 52 & NR & NR & 13 & 1 & 31 & 20 & 9 & 3 & 4 & 4 & 4.0 \\
\hline Maeda [24] & 2017 & $\mathrm{BC}$ & 47 & 10 & 0 & 23 & 4 & 47 & 25 & 38 & 16 & 4 & 4 & 3.0 \\
\hline Takashima [25] & 2016 & $\mathrm{BC}$ & 35 & 18 & 0 & 18 & 0 & 34 & 22 & 31 & 9 & 2 & 2 & 4.0 \\
\hline Yardley [26] & 2016 & $\mathrm{BC}$ & 65 & NR & NR & 16 & 3 & 29 & 24 & 8 & 7 & NR & NR & 4.0 \\
\hline Garrone [27] & 2016 & $\mathrm{BC}$ & 113 & 8 & 1 & 32 & 3 & 41 & 22 & 19 & 5 & 1 & 1 & 4.0 \\
\hline Dell'Ova [28] & 2015 & $\mathrm{BC}$ & 258 & 27 & 1 & NR & 4 & 99 & 54 & NR & NR & NR & 13 & 4.03 \\
\hline Smith [29] & 2016 & $\mathrm{BC}$ & 67 & NR & NR & NR & NR & 5 & 5 & NR & NR & 1 & 1 & NR \\
\hline Wilks [30] & 2014 & $\mathrm{BC}$ & 52 & NR & NR & 13 & 1 & 31 & 20 & 9 & 3 & 4 & 4 & 4.0 \\
\hline Gitlitz [31] & 2012 & NSCLC & 66 & 7 & 1 & 43 & 0 & 41 & 36 & 41 & 19 & NR & NR & 3.0 \\
\hline Dranitsaris [32] & 2015 & $\mathrm{BC}$ & 90 & 7 & NR & 28 & NR & 29 & NR & NR & NR & 8 & NR & NR \\
\hline Fabi [33] & 2015 & $\mathrm{BC}$ & 78 & NR & NR & NR & 2 & NR & 17 & NR & 24 & NR & NR & 4.02 \\
\hline Hensley [34] & 2011 & $\mathrm{OC}$ & 73 & NR & NR & NR & 1 & 35 & NR & 23 & NR & 1 & NR & NR \\
\hline Ates [35] & 2016 & $\mathrm{BC}$ & 66 & 3 & 2 & NR & NR & 25 & 16 & NR & NR & NR & $\mathrm{NR}$ & 4.0 \\
\hline Kawai [36] & 2017 & STS & 51 & NR & NR & 24 & 7 & 50 & 44 & 51 & 38 & NR & NR & 4.0 \\
\hline Kaklamani [37] & 2015 & $\mathrm{BC}$ & 30 & 24 & 6 & 30 & 7 & 23 & 18 & NR & NR & NR & NR & 4.0 \\
\hline Kessler [38] & 2015 & $\mathrm{BC}$ & 48 & NR & NR & NR & NR & 13 & 9 & NR & NR & NR & NR & 4.0 \\
\hline Lorusso [39] & 2017 & $\mathrm{BC}$ & 91 & 4 & 0 & 15 & 2 & 27 & 11 & NR & NR & NR & NR & NR \\
\hline McIntyre [40] & 2014 & $\mathrm{BC}$ & 56 & NR & NR & 20 & 2 & 40 & 28 & 19 & 12 & NR & NR & 4.0 \\
\hline Gamucci [41] & 2014 & $\mathrm{BC}$ & 133 & 11 & 3 & 19 & 1 & 38 & 19 & NR & NR & NR & $\mathrm{NR}$ & 4.0 \\
\hline Moscetti [42] & 2017 & $\mathrm{BC}$ & 50 & NR & NR & 16 & 0 & 33 & 2 & NR & NR & NR & NR & 4.0 \\
\hline Prestifilippo [43] & 2017 & $\mathrm{BC}$ & 31 & NR & NR & 15 & 2 & NR & NR & 18 & 0 & 15 & 8 & NR \\
\hline Quaquarini [44] & 2017 & $\mathrm{BC}$ & 44 & 3 & 2 & 19 & 2 & 23 & 5 & 22 & 4 & 1 & 1 & 4.02 \\
\hline Inoue [45] & 2016 & $\mathrm{BC}$ & 51 & 2 & 0 & 15 & 3 & 29 & 18 & 30 & 12 & 4 & 4 & 4.0 \\
\hline Schoffski [46] & 2011 & STS & 127 & NR & NR & 112 & 9 & 101 & 66 & 107 & 44 & NR & NR & 3.0 \\
\hline Vahdat [47] & 2009 & $\mathrm{BC}$ & 33 & 1 & 0 & 5 & 0 & 23 & 20 & 5 & 5 & 1 & 1 & 3.0 \\
\hline
\end{tabular}

Abbreviations: CTCAE, common terminology criteria for adverse events; BC, breast cancer; HNC, head and neck cancer; NSCLC, non-small cell lung cancer; OC, ovarian cancer; PC, prostate cancer, STS, soft tissue sarcoma; NR, not reported.

1 (TGF- $\beta 1$ ), which linked to multiple gene that controlled the growth, proliferation, differentiation and apoptosis of blood cells [50]. Our results were consistent with pre-clinical discoveries and corroborated the theory that eribulin treatment increased the risk of myelosuppression in cancer.
Five types of hematologic toxicities were analyzed in the present study. Interestingly, high incidence rates and risks only occurred in two types of hematologic AEs, neutropenia and leucopenia. The incidence rates and risks of other two types of hematologic toxicities, anemia and febrile neutropenia, did not show significant 


\section{Neutropenia}

\begin{tabular}{|c|c|c|c|c|c|c|c|}
\hline \multirow[b]{2}{*}{ Study } & \multicolumn{2}{|c|}{ Eribulin } & \multicolumn{2}{|c|}{ Control } & & \multirow[b]{2}{*}{ Risk Ratio, $95 \% \mathrm{Cl}$} & \multirow[b]{2}{*}{ Weight, $\%$} \\
\hline & Total & Events & Total & Events & & & \\
\hline Cortes (2011) & 508 & 260 & 254 & 73 & & $1.78(1.44,2.20)$ & 22.80 \\
\hline abraham (2015) & 30 & 7 & 19 & 2 & & $2.22(0.51,9.57)$ & 4.59 \\
\hline Kaufman (2015) & 554 & 295 & 548 & 87 & $\rightarrow$ & $3.35(2.72,4.13)$ & 22.87 \\
\hline Vahdat (2013) & 51 & 24 & 50 & 14 & & $1.68(0.99,2.86)$ & 15.73 \\
\hline Schoffski (2016) & 228 & 99 & 224 & 53 & & $1.84(1.39,2.42)$ & 21.48 \\
\hline Waller (2015) & 41 & 12 & 39 & 11 & & $1.04(0.52,2.07)$ & 12.54 \\
\hline \multicolumn{5}{|c|}{ Overall $\left(I^{2}=81.5 \%, p=0.00\right)$} & & $1.94(1.37,2.74)$ & 100.00 \\
\hline
\end{tabular}

\section{Leucopenia}

\begin{tabular}{|c|c|c|c|c|c|c|c|c|}
\hline Cortes (2011) & 508 & 116 & 254 & 28 & & & $2.07(1.41,3.04)$ & 29.85 \\
\hline Kaufman (2015) & 554 & 171 & 548 & 57 & & $\longrightarrow$ & $2.97(2.25,3.91)$ & 32.71 \\
\hline Schoffski (2016) & 228 & 36 & 224 & 23 & & & $1.54(0.94,2.51)$ & 26.84 \\
\hline Waller (2015) & 41 & 3 & 39 & 7 & $\leftarrow$ & & $0.41(0.11,1.47)$ & 10.61 \\
\hline \multicolumn{6}{|c|}{ Overall $\left(I^{2}=77.3 \%, p=0.01\right)$} & & $1.81(1.10,2.97)$ & 100.00 \\
\hline
\end{tabular}

Anaemia

\begin{tabular}{|c|c|c|c|c|c|c|c|}
\hline Cortes (2011) & 508 & 94 & 254 & 56 & $\rightarrow$\begin{tabular}{l|l}
0 \\
\hdashline
\end{tabular} & $0.84(0.63,1.13)$ & 28.04 \\
\hline Kaufman (2015) & 554 & 104 & 548 & 96 & & $1.07(0.83,1.38)$ & 36.25 \\
\hline Vahdat (2013) & 51 & 13 & 50 & 10 & & $1.27(0.62,2.63)$ & 3.79 \\
\hline Schoffski (2016) & 228 & 67 & 224 & 69 & & $0.95(0.72,1.26)$ & 26.14 \\
\hline Waller (2015) & 41 & 8 & 39 & 15 & $\leftarrow$ & $0.51(0.24,1.06)$ & 5.77 \\
\hline \multicolumn{6}{|c|}{ Overall $\left(I^{2}=19.6 \%, p=0.29\right)$} & $0.95(0.82,1.11)$ & 100.00 \\
\hline
\end{tabular}

\section{Febrile Neutropenia}

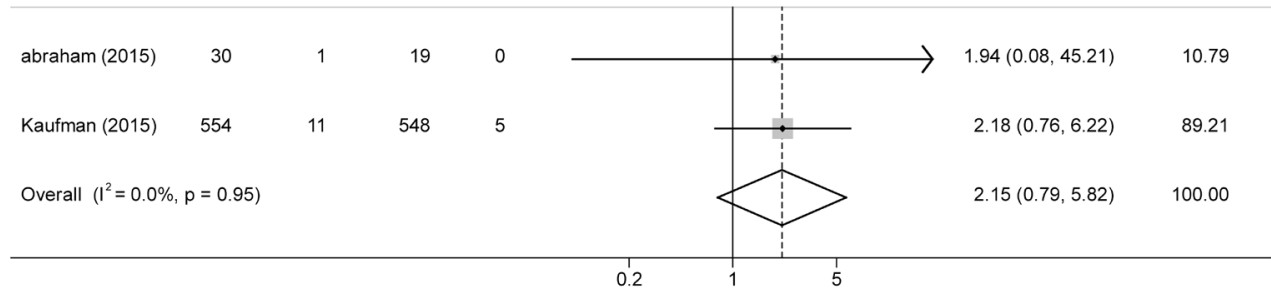

Thrombocytopenia

\begin{tabular}{llllll|l}
\hline Schoffski(2016) & 228 & 13 & 224 & 62 & $0.21(0.12,0.36)$ \\
$0.21(0.12,0.36)$ & \\
\hline
\end{tabular}

Figure 2: Forest plots of relative risk (RR) of all-grade hematologic toxicities associated with eribulin mesylate versus control in cancer patients. 
Neutropenia

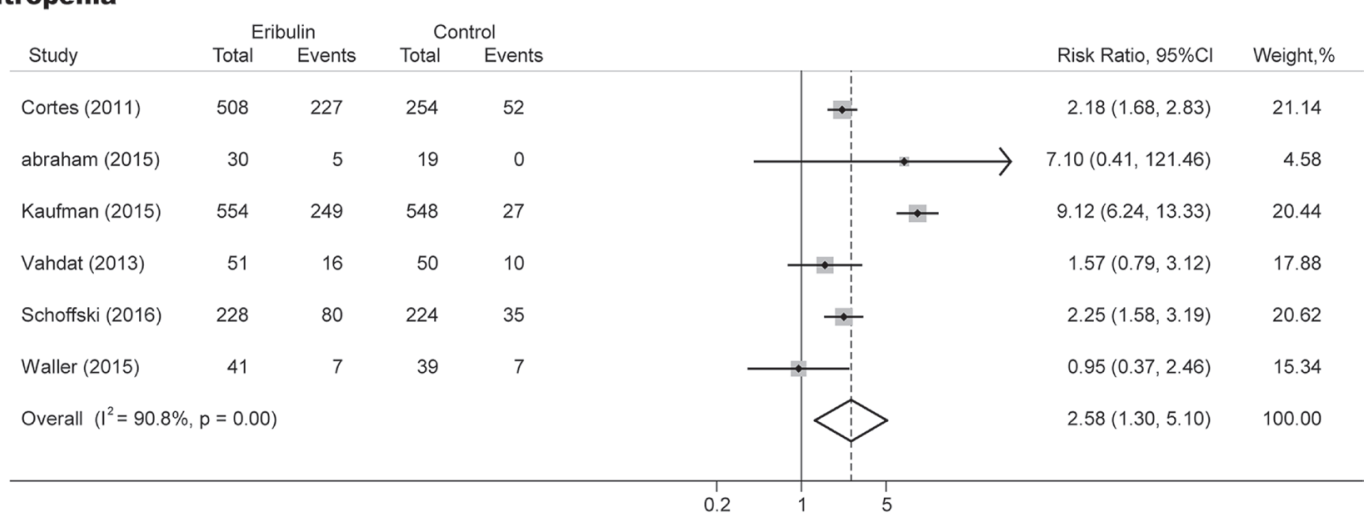

\section{Leucopenia}

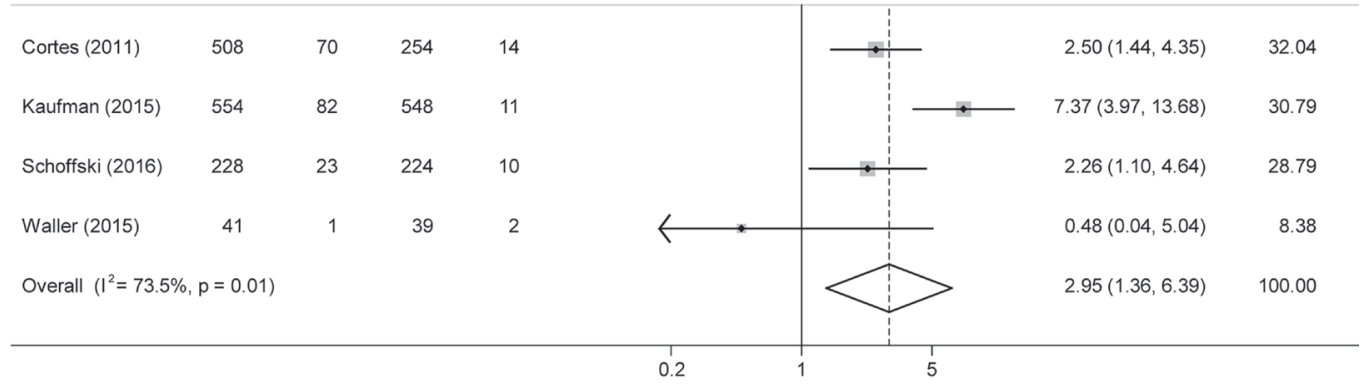

\section{Anaemia}

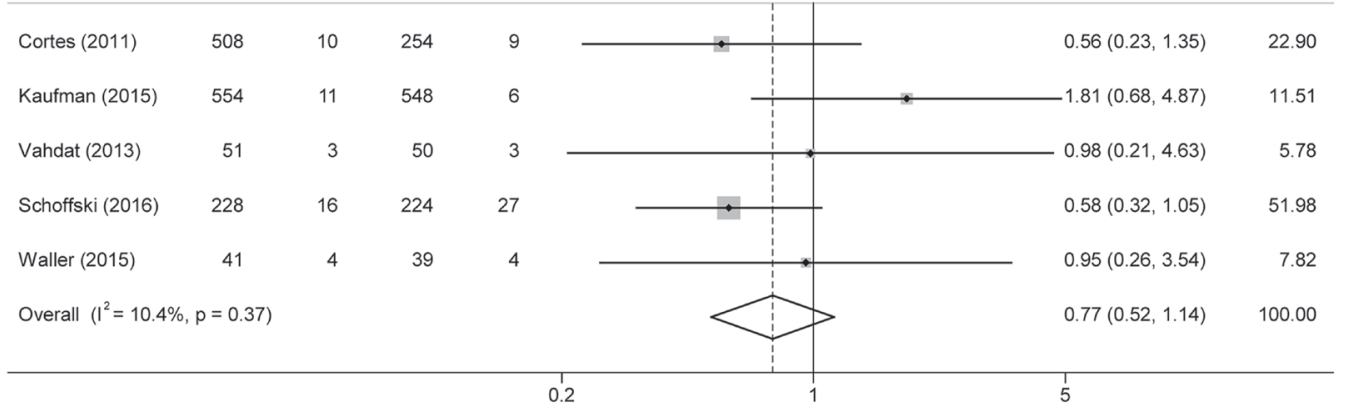

Febrile Neutropenia

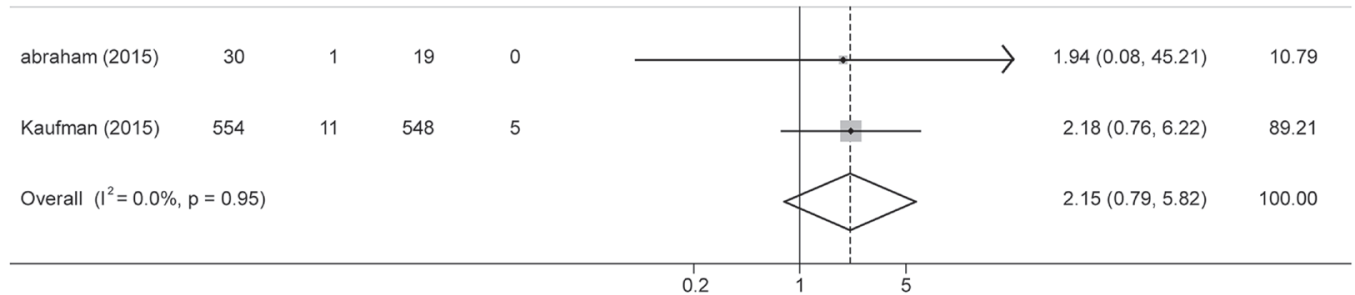

Thrombocytopenia

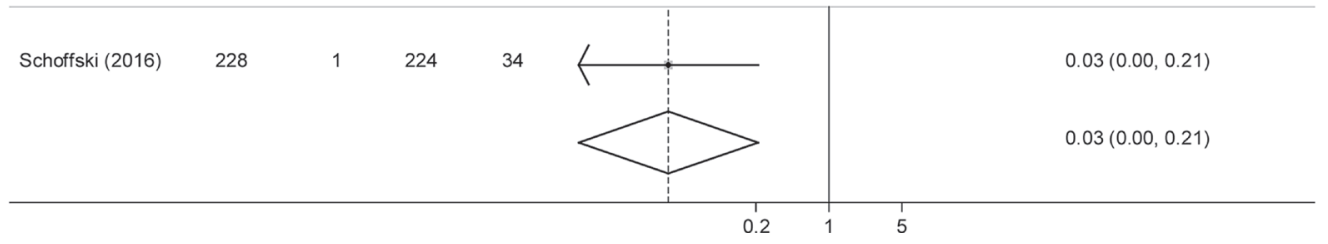

Figure 3: Forest plots of relative risk of high-grade hematologic toxicities associated with eribulin mesylate versus control in cancer patients. 


\section{Neutropenia}

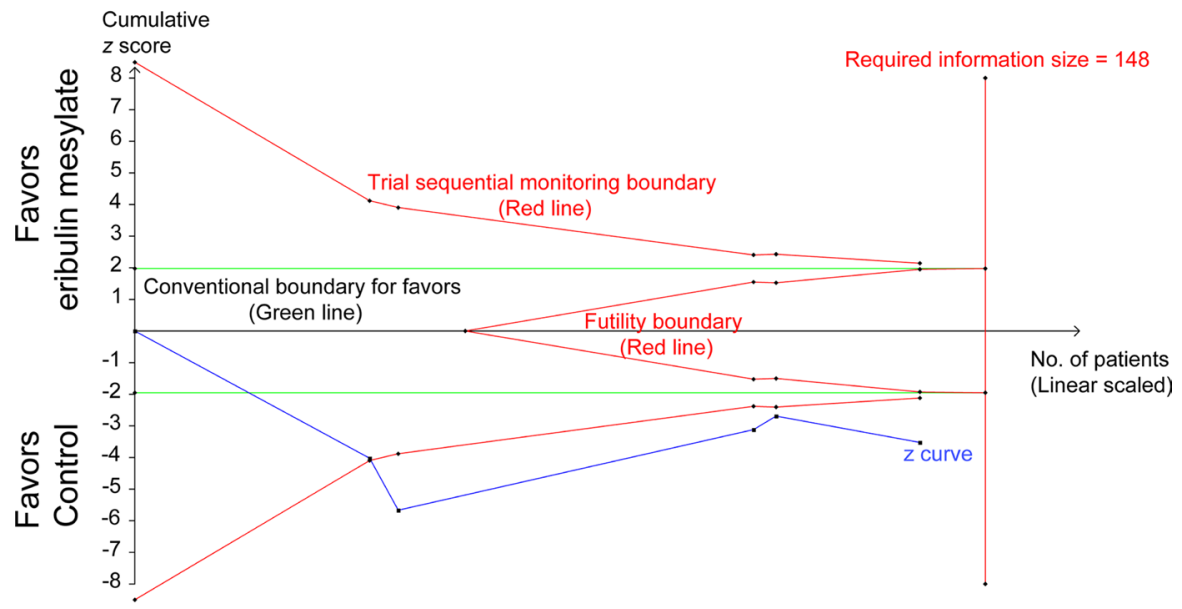

\section{Leucopenia}

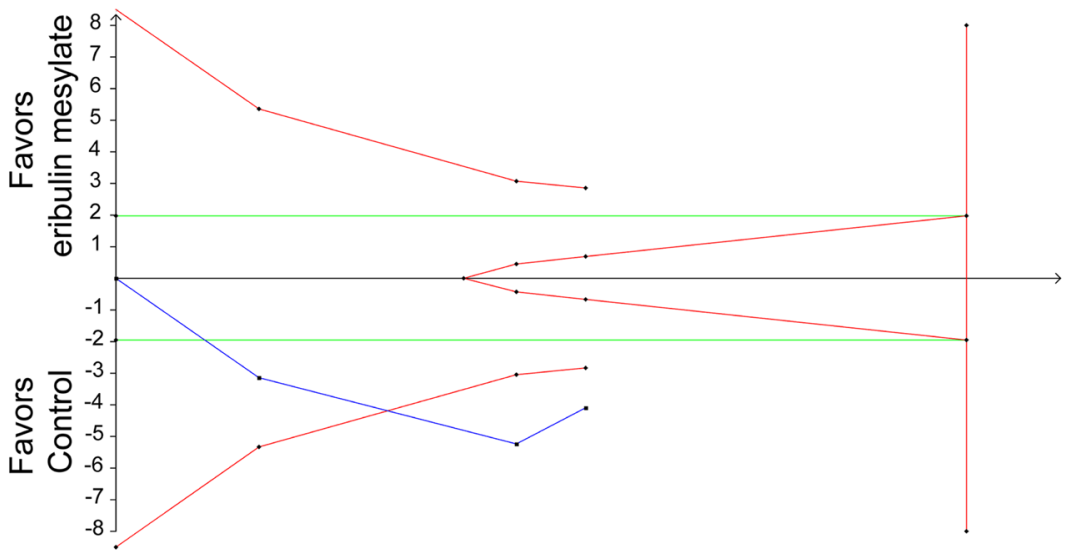

Anemia

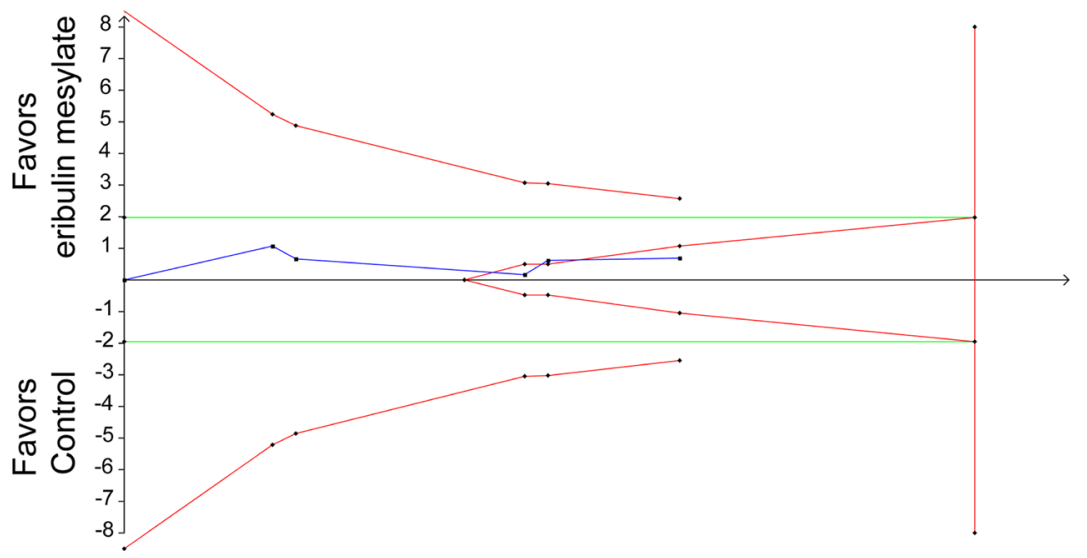

Figure 4: Trial sequential analysis (TSA) of eligible studies comparing eribulin therapy with control. The cumulative $z$ curve crossed both the conventional boundary and the trial sequential monitoring boundary in neutropenia and leucopenia analysis, crossed the futility boundary and entered the futility area in anemia analysis; establishing sufficient and conclusive evidence and suggesting that further studies were not required. $\mathrm{X}$ axis, Number of patients randomized; $\mathrm{Y}$ axis, cumulative $z$ score; horizontal green lines, conventional boundaries (upper line, $z$ score $=1.96$; lower line, $z$ score $=-1.96$; two sided $p=0.05$ ); sloping red lines with black filled square, trial sequential monitoring boundaries; blue line with black filled squares, $z$ curve; vertical red line, required information size. 
Table 3: Overall incidences of all-grade hematologic toxicities in patients treated with eribulin

\begin{tabular}{lccccc}
\hline Hematologic toxicities & No. of trials & No. of patients & Incidence (95\% CI) & $\left.\boldsymbol{I}^{\mathbf{2}} \mathbf{\%}\right)$ & $\boldsymbol{p}$ \\
\hline Neutropenia & 37 & 4,849 & $56 \%(46 \%-65 \%)$ & 98.7 & 0.00 \\
BC & 29 & 4,118 & $56 \%(45 \%-66 \%)$ & 98.8 & 0.00 \\
Non-BC & 8 & 731 & $55 \%(34 \%-76 \%)$ & 98.2 & 0.00 \\
Leucopenia & 26 & 3,825 & $44 \%(30 \%-59 \%)$ & 99.4 & 0.00 \\
BC & 18 & 3,094 & $44 \%(26 \%-61 \%)$ & 99.4 & 0.00 \\
Non-BC & 8 & 731 & $45 \%(13 \%-77 \%)$ & 99.5 & 0.00 \\
Anemia & 31 & 4,258 & $33 \%(25 \%-40 \%)$ & 97.3 & 0.00 \\
BC & 24 & 3,600 & $27 \%(22 \%-32 \%)$ & 93.1 & 0.00 \\
Non-BC & 7 & 658 & $47 \%(24 \%-70 \%)$ & 97.8 & 0.00 \\
Febrile neutropenia & 18 & 2,760 & $5 \%(3 \%-7 \%)$ & 80.0 & 0.00 \\
BC & 16 & 2,582 & $6 \%(3 \%-8 \%)$ & 81.8 & 0.00 \\
Non-BC & 2 & 178 & $2 \%(0 \%-5 \%)$ & 10.3 & 0.29 \\
Thrombocytopenia & 18 & 2,512 & $12 \%(8 \%-15 \%)$ & 90.9 & 0.00 \\
BC & 15 & 2,113 & $13 \%(9 \%-17 \%)$ & 92.4 & 0.00 \\
Non-BC & 3 & 399 & $7 \%(4 \%-9 \%)$ & 0.0 & 0.45 \\
\hline
\end{tabular}

Table 4: Overall incidences of high-grade hematologic toxicities in patients treated with eribulin

\begin{tabular}{lccccc}
\hline Hematologic toxicities & No. of trials & No. of patients & Incidence (95\% CI) & $\boldsymbol{I}^{\mathbf{2}} \mathbf{( \% )}$ & $\boldsymbol{p}$ \\
\hline Neutropenia & 36 & 4,764 & $39 \%(30 \%-48 \%)$ & 98.2 & 0.00 \\
BC & 29 & 4,106 & $39 \%(29 \%-49 \%)$ & 98.4 & 0.00 \\
Non-BC & 7 & 658 & $41 \%(23 \%-59 \%)$ & 96.4 & 0.00 \\
Leucopenia & 26 & 3,830 & $21 \%(14 \%-28 \%)$ & 97.2 & 0.00 \\
BC & 19 & 3,172 & $20 \%(12 \%-28 \%)$ & 97.5 & 0.00 \\
Non-BC & 7 & 658 & $24 \%(14 \%-28 \%)$ & 96.2 & 0.00 \\
Anemia & 33 & 4,577 & $2 \%(1 \%-3 \%)$ & 41.7 & 0.01 \\
BC & 25 & 3,846 & $2 \%(2 \%-3 \%)$ & 23.2 & 0.15 \\
Non-BC & 8 & 731 & $4 \%(2 \%-6 \%)$ & 68.7 & 0.00 \\
Febrile neutropenia & 17 & 2,855 & $5 \%(3 \%-6 \%)$ & 74.8 & 0.00 \\
BC & 16 & 2,750 & $5 \%(3 \%-7 \%)$ & 76.4 & 0.00 \\
Non-BC & 1 & 105 & $4 \%(0 \%-7 \%)$ & NA & NA \\
Thrombocytopenia & 17 & 2,422 & $12 \%(6 \%-18 \%)$ & 37.0 & 0.06 \\
BC & 14 & 2,023 & $13 \%(6 \%-21 \%)$ & 43.3 & 0.04 \\
Non-BC & 3 & 399 & $6 \%(0 \%-14 \%)$ & 0.0 & 0.49 \\
\hline BC, bea
\end{tabular}

$\mathrm{BC}$, breast cancer.

difference compared with control. The incidences and relative risk of eribulin related thrombocytopenia needed further investigation. These discrepancies might be due to the differences in the mechanisms of action among these hematologic toxicities [48]. Hematologic events were one of the most common adverse events that could lead to therapy adjustment and discontinuation in clinical trials. High-grade neutropenia and leucopenia were often clinically significant and needed careful medical intervention considering these AEs potentially led to hemorrhage and sepsis in patients. In fact, some large RCTs even reported that fatal adverse events occurred because of hematologic toxicities [9, 11, 13]. During eribulin treatment, patients who developed neutropenia and leucopenia were usually managed with dose reduction, treatment delays and granulocyte colonystimulating factor (G-CSF). The application of G-CSF to manage eribulin related high-grade neutropenia was at the physician's choice in accordance with relevant clinical practice guidelines such as European Society for Medical 
Table 5: Relative risk of neutropenia and leucopenia excluded one study [11]

\begin{tabular}{ccccc}
\hline & Hematologic toxicities & RR (95\% CI) & $\boldsymbol{I}^{\mathbf{2}}$ & $\boldsymbol{p}$ \\
\hline \multirow{2}{*}{ All-grade } & Neutropenia & $1.75(1.50-2.04)$ & $0.0 \%$ & 0.65 \\
& Leucopenia & $1.71(1.28-2.29)$ & $36.0 \%$ & 0.05 \\
\multirow{2}{*}{ High-grade } & Neutropenia & $2.10(1.73-2.56)$ & $6.7 \%$ & 0.37 \\
& Leucopenia & $2.29(1.49-3.51)$ & $0.0 \%$ & 0.40 \\
\hline
\end{tabular}

Oncology (ESMO) [51] and European Organization for Research and Treatment of Cancer (EORTC) [52]. It should be noted that neutropenia was reported at a higher incidence in the Asian population than that in the Western patients partly because of the pharmacogenomics [18].

Since we cannot access to any individual patient data, we did not correlate the risks of infection, bleeding and mortality with neutropenia and leucopenia. Although previous studies reported that poor performance status, advanced age, and low baseline blood cell counts were significant predictors for the severity of neutropenia [53], there were still no appropriate technique to determine which patients were at high risk of neutropenia and leucopenia. Accordingly, regular monitoring was essential for patients treated with eribulin.

Here we conducted a comprehensive review using the most up-to-date published data, which made our results more extensive. Moreover, with the accumulating evidence and enlarged sample size, we had enhanced the statistical power to provide more precise and reliable estimates. However, our study was restricted by some limitations. First, this was a meta-analysis conducted at the trial level and no clinicopathological variable at the patient level could be analyzed. Second, pooled incidence rates had significant heterogeneities, and this might be due to the different types of underlying malignancies, sample size, insufficient follow-up data among the included trials.

In conclusion, our meta-analysis revealed that eribulin mesylate was associated with an increased risk of hematological toxicities compared with controls. Clinical doctors should be acknowledged of these potential adverse events and hematologic monitoring at regular intervals may be advised.

\section{MATERIALS AND METHODS}

The present study was reported according to the Preferred Reporting Items for Systematic Reviews and Meta-Analyses (PRISMA) statement [54].

\section{Literature search and study selection}

A systematic search of Embase and PubMed database from January 1966 to August 2017 was conducted without any language restrictions. The only search keyword and Medical subject heading used was eribulin. Both inclusion and exclusion criteria were prespecified. To be eligible, published studies had to meet the following criteria: (1) population: non-phase I trials in patients $(n>30)$ with solid tumor; (2) intervention: eribulin was administered at a standard dose of $1.4 \mathrm{mg} /$ $\mathrm{m}^{2}$ in 2-5 minutes intravenously on days 1 and 8 in a 21-day schedule, the currently FDA-recommended dose until unacceptable toxicity, disease progression or patient refusal; (3) clinical outcomes: events or events rates and sample size information for both all-grade and high-grade hematologic toxicities including neutropenia, leucopenia, anemia, febrile neutropenia and thrombocytopenia. Phase I trials were not included because of the small size of patients and various doses in these studies. Other publications on this topic, including review articles, conference abstract, pre-clinical papers, early versions of data later published, editorials, articles not dealing with eribulin were not included (Figure 1). Considering recent progress with eribulin had not been published, electronic searches were also carried out in two major international congresses' proceedings (American Society of Clinical Oncology Annual Meeting and European Society of Medical Oncology). When multiple publications of the same study occurred, only the most recent and/or most complete reporting study was included. Any discrepancies were settled by discussion and consensus. Two authors independently conducted the initial search, screened the titles and abstracts, and classified trials as excluded, included and uncertain. Any discrepancy was resolved by consensus.

\section{Data extraction}

Eligible abstracts were collected and full texts of relevant articles were checked for the trial design and reporting of hematologic toxicities. The following items were extracted: name of the first author, region, year of publication, underlying malignancy, number of patients enrolled, median age, gender, European cooperative oncology group performance status (ECOG PS), median treatment duration, median overall survival (OS), median progression-free survival (PFS) (Table 1), numbers of events of the following AEs (for both all-grade and high-grade): neutropenia, leucopenia, anemia, febrile neutropenia and thrombocytopenia (Table 2). The number of subjects evaluated for toxicity was used as the number analyzed for each study, unless it was indicated otherwise. 


\section{Quality assessment of the eligible studies}

Newcastle-Ottawa Scale (NOS) was conducted to assess the methodological quality of all included studies [55]. This scale used a star system to evaluate any study in three sections: selection of participants, comparability of study groups, and the ascertainment of outcomes of interest.

\section{Statistical analysis}

The primary analysis examined the overall incidence, relative risk and corresponding $95 \% \mathrm{CI}$ of allgrade and high-grade hematologic toxicities in cancer patients treated by eribulin. To calculate the incidence, the number of patients receiving eribulin and the number of hematologic toxicities were extracted from the eligible single-arm and randomized controlled trials. The proportion of patients with neutropenia, leucopenia, anemia, febrile neutropenia and thrombocytopenia and 95\% CIs were derived from each trial. We calculated RRs and their CIs with data extracted from RCTs only, comparing the incidence of each adverse event in patients assigned to eribulin arms with patients in placebo or control arms. Statistical heterogeneity between different trials or subgroups was evaluated by Cochrane's Q statistic. The $I^{2}$ statistic was calculated to assess the extent of inconsistency contributable to the heterogeneity across different studies [56]. The assumption of homogeneity was considered invalid for $I^{2}>25 \%$ or $p<0.05$. Summary RRs and incidences were calculated using fixed-effects or random-effects models depending on the heterogeneity of included trials. Potential publication bias was assessed by visual inspection of a funnel plot, and also evaluated using the tests of Egger et al. [57] and Begg et al. [58]. Twosided $p<0.05$ were considered statistically significant. All analysis was performed using Stata version 12.0 (StataCorp, USA).

\section{Trial sequential analysis}

Random errors increase the risk of type I error (false-positive results) in meta-analysis because of sparse data and/or repetitive examining $[59,60]$. As a result, trial sequential monitoring boundaries, namely trial sequential analysis (TSA), were conducted [59, 61, 62]. It can determine whether the data in any meta-analysis is reliable and conclusive. When the cumulative $z$ curve crosses the trial sequential monitoring boundary or enters the futility area, a sufficient level of evidence for the anticipated intervention effect may have been reached and no further trials are needed. If the $z$ curve crosses none of the boundaries and the required information size has not been reached, there is insufficient evidence to reach a conclusion. Here, we estimated the required information size using $\alpha=0.05$ (two-sided), $\beta=0.20$ (power of $80 \%$ ).
All analysis was conducted by TSA version 0.9.5.9 Beta (http://www.ctu.dk/tsa).

\section{CONFLICTS OF INTEREST}

None.

\section{FUNDING}

This work was funded by National Natural Science Foundation of China (No. 31571417 and No. 81502478), Natural Science Foundation of Heilongjiang Province (No. H2016023) and Haiyan Foundation of the Third Affiliated Hospital of Harbin Medical University (No. JJMS201406).

\section{REFERENCES}

1. Swami U, Chaudhary I, Ghalib MH, Goel S. Eribulin -- a review of preclinical and clinical studies. Crit Rev Oncol Hematol. 2012; 81:163-84. https://doi.org/10.1016/j. critrevonc.2011.03.002.

2. Mani S, Swami U. Eribulin mesilate, a halichondrin $\mathrm{B}$ analogue, in the treatment of breast cancer. Drugs Today (Barc). 2010; 46:641-53. https://doi.org/10.1358/ dot.2010.46.9.1519020.

3. Jordan MA, Kamath K, Manna T, Okouneva T, Miller HP, Davis C, Littlefield BA, Wilson L. The primary antimitotic mechanism of action of the synthetic halichondrin E7389 is suppression of microtubule growth. Mol Cancer Ther. 2005; 4:1086-95. https://doi.org/10.1158/1535-7163.mct-04-0345.

4. Yoshida T, Ozawa Y, Kimura T, Sato Y, Kuznetsov G, Xu S, Uesugi M, Agoulnik S, Taylor N, Funahashi Y, Matsui J. Eribulin mesilate suppresses experimental metastasis of breast cancer cells by reversing phenotype from epithelialmesenchymal transition (EMT) to mesenchymal-epithelial transition (MET) states. Br J Cancer. 2014; 110:1497-505. https://doi.org/10.1038/bjc.2014.80.

5. Funahashi Y, Okamoto K, Adachi Y, Semba T, Uesugi M, Ozawa Y, Tohyama O, Uehara T, Kimura T, Watanabe H, Asano M, Kawano S, Tizon X, et al. Eribulin mesylate reduces tumor microenvironment abnormality by vascular remodeling in preclinical human breast cancer models. Cancer Sci. 2014; 105:1334-42. https://doi.org/10.1111/ cas. 12488 .

6. Jain S, Vahdat LT. Eribulin mesylate. Clin Cancer Res. 2011; 17:6615-22. https://doi.org/10.1158/1078-0432.ccr11-1807.

7. Osgood CL, Chuk MK, Theoret MR, Huang L, He K, Her L, Keegan P, Pazdur R. FDA Approval Summary: Eribulin for Patients with Unresectable or Metastatic Liposarcoma who have Received a Prior Anthracycline-Containing Regimen. Clin Cancer Res. 2017; 23:6384-6389. https:// doi.org/10.1158/1078-0432.ccr-16-2422. 
8. Swami U, Shah U, Goel S. Eribulin in Cancer Treatment. Mar Drugs. 2015; 13:5016-58. https://doi.org/10.3390/ md13085016.

9. Cortes J, O'Shaughnessy J, Loesch D, Blum JL, Vahdat LT, Petrakova K, Chollet P, Manikas A, Dieras V, Delozier T, Vladimirov V, Cardoso F, Koh H, et al. Eribulin monotherapy versus treatment of physician's choice in patients with metastatic breast cancer (EMBRACE): a phase 3 open-label randomised study. Lancet. 2011; 377:914-23. https://doi.org/10.1016/s0140-6736(11)60070-6.

10. Abraham J, Robidoux A, Tan AR, Limentani S, Sturtz K, Shalaby I, Alcorn H, Buyse ME, Wolmark N, Jacobs SA. Phase II randomized clinical trial evaluating neoadjuvant chemotherapy regimens with weekly paclitaxel or eribulin followed by doxorubicin and cyclophosphamide in women with locally advanced HER2-negative breast cancer: NSABP Foundation Study FB-9. Breast Cancer Res Treat. 2015; 152:399-405. https://doi.org/10.1007/s10549-0153466-4.

11. Kaufman PA, Awada A, Twelves C, Yelle L, Perez EA, Velikova G, Olivo MS, He Y, Dutcus CE, Cortes J. Phase III open-label randomized study of eribulin mesylate versus capecitabine in patients with locally advanced or metastatic breast cancer previously treated with an anthracycline and a taxane. J Clin Oncol. 2015; 33:594-601. https://doi. org/10.1200/jco.2013.52.4892.

12. Vahdat LT, Garcia AA, Vogel C, Pellegrino C, Lindquist DL, Iannotti N, Gopalakrishna P, Sparano JA. Eribulin mesylate versus ixabepilone in patients with metastatic breast cancer: a randomized Phase II study comparing the incidence of peripheral neuropathy. Breast Cancer Res Treat. 2013; 140:341-51. https://doi.org/10.1007/s10549013-2574-2.

13. Schoffski P, Chawla S, Maki RG, Italiano A, Gelderblom H, Choy E, Grignani G, Camargo V, Bauer S, Rha SY, Blay JY, Hohenberger P, D'Adamo D, et al. Eribulin versus dacarbazine in previously treated patients with advanced liposarcoma or leiomyosarcoma: a randomised, open-label, multicentre, phase 3 trial. Lancet. 2016; 387:1629-37. https://doi.org/10.1016/s0140-6736(15)01283-0.

14. Waller CF, Vynnychenko I, Bondarenko I, Shparyk Y, Hodge JP, Freeman A, Huber B, Lieberman R, Shelton MJ, Dave H. An open-label, multicenter, randomized phase Ib/ II study of eribulin mesylate administered in combination with pemetrexed versus pemetrexed alone as second-line therapy in patients with advanced nonsquamous non-smallcell lung cancer. Clin Lung Cancer. 2015; 16:92-9. https:// doi.org/10.1016/j.cllc.2014.10.001.

15. Watanabe J, Ito $\mathrm{Y}$, Ohsumi S, Mizutani M, Tashiro H, Sakurai K, Takahashi M, Saito T, Tsurutani J, Mukai H, Yoshinami T, Takao S, Yamamoto Y, et al. Safety and effectiveness of eribulin in Japanese patients with locally advanced or metastatic breast cancer: a post-marketing observational study. Invest New Drugs. 2017; 35:791-799. https://doi.org/10.1007/s10637-017-0486-4.
16. de Bono JS, Molife LR, Sonpavde G, Maroto JP, Calvo E, Cartwright TH, Loesch DM, Feit K, Das A, Zang EA, Wanders J, Agoulnik S, Petrylak DP. Phase II study of eribulin mesylate (E7389) in patients with metastatic castration-resistant prostate cancer stratified by prior taxane therapy. Ann Oncol. 2012; 23:1241-9. https://doi. org/10.1093/annonc/mdr380.

17. Aftimos P, Polastro L, Ameye L, Jungels C, Vakili J, Paesmans M, van den Eerenbeemt J, Buttice A, Gombos A, de Valeriola D, Gil T, Piccart-Gebhart M, Awada A. Results of the Belgian expanded access program of eribulin in the treatment of metastatic breast cancer closely mirror those of the pivotal phase III trial. Eur J Cancer. 2016; 60:117-24. https://doi.org/10.1016/j.ejca.2016.03.010.

18. Aogi K, Iwata H, Masuda N, Mukai H, Yoshida M, Rai Y, Taguchi K, Sasaki Y, Takashima S. A phase II study of eribulin in Japanese patients with heavily pretreated metastatic breast cancer. Ann Oncol. 2012; 23:1441-8. https://doi.org/10.1093/annonc/mdr444.

19. Arnold SM, Moon J, Williamson SK, Atkins JN, Ou SH, LeBlanc M, Urba SG. Phase II evaluation of eribulin mesylate (E7389, NSC 707389) in patients with metastatic or recurrent squamous cell carcinoma of the head and neck: Southwest Oncology Group trial S0618. Invest New Drugs. 2011; 29:352-9. https://doi.org/10.1007/s10637-009-9348-z.

20. Cortes J, Vahdat L, Blum JL, Twelves C, Campone M, Roche H, Bachelot T, Awada A, Paridaens R, Goncalves A, Shuster DE, Wanders J, Fang F, et al. Phase II study of the halichondrin B analog eribulin mesylate in patients with locally advanced or metastatic breast cancer previously treated with an anthracycline, a taxane, and capecitabine. J Clin Oncol. 2010; 28:3922-8. https://doi.org/10.1200/ jco.2009.25.8467.

21. Park YH, Kim TY, Im YH, Lee KS, Park IH, Sohn J, Lee SH, Im SA, Kim JH, Kim SH, Lee SJ, Koh SJ, Lee KH, et al. Feasibility and Efficacy of Eribulin Mesilate in Korean Patients with Metastatic Breast Cancer: Korean Multicenter Phase IV Clinical Study Results. Cancer Res Treat. 2017; 49:423-9. https://doi.org/10.4143/crt.2016.191.

22. Araki K, Fukada I, Yanagi H, Kobayashi K, Shibayama T, Horii R, Takahashi S, Akiyama F, Ohno S, Ito Y. First report of eribulin in combination with pertuzumab and trastuzumab for advanced HER2-positive breast cancer. Breast. 2017; 35:78-84. https://doi.org/10.1016/j. breast.2017.06.015.

23. Puhalla S, Wilks S, Brufsky AM, O’Shaughnessy J, Schwartzberg LS, Berrak E, Song J, Vahdat L. Clinical effects of prior trastuzumab on combination eribulin mesylate plus trastuzumab as first-line treatment for human epidermal growth factor receptor 2 positive locally recurrent or metastatic breast cancer: results from a Phase II, singlearm, multicenter study. Breast Cancer (Dove Med Press). 2016; 8:231-9. https://doi.org/10.2147/bctt.s98696.

24. Maeda S, Saimura M, Minami S, Kurashita K, Nishimura R, Kai Y, Yano H, Mashino K, Mitsuyama S, Shimokawa 
M, Tamura K. Efficacy and safety of eribulin as first- to third-line treatment in patients with advanced or metastatic breast cancer previously treated with anthracyclines and taxanes. Breast. 2017; 32:66-72. https://doi.org/10.1016/j. breast.2016.12.017.

25. Takashima T, Tokunaga S, Tei S, Nishimura S, Kawajiri H, Kashiwagi S, Yamagata S, Noda S, Nishimori T, Mizuyama Y, Sunami T, Tezuka K, Ikeda K, et al. A phase II, multicenter, single-arm trial of eribulin as first-line chemotherapy for HER2-negative locally advanced or metastatic breast cancer. Springerplus. 2016; 5:164. https:// doi.org/10.1186/s40064-016-1833-1.

26. Yardley DA, Reeves J, Dees EC, Osborne C, Paul D, Ademuyiwa F, Soliman H, Guthrie T, Andersen J, Krekow L, Choksi J, Daniel B, Danso M, et al. Ramucirumab With Eribulin Versus Eribulin in Locally Recurrent or Metastatic Breast Cancer Previously Treated With Anthracycline and Taxane Therapy: A Multicenter, Randomized, Phase II Study. Clin Breast Cancer. 2016; 16:471-9.e1. https://doi. org/10.1016/j.clbc.2016.07.005.

27. Garrone O, Montemurro F, Saggia C, La Verde N, Vandone AM, Airoldi M, De Conciliis E, Donadio M, Lucio F, Polimeni MA, Oletti MV, Giacobino A, Merlano MC. Eribulin in pretreated metastatic breast cancer patients: results of the TROTTER trial-a multicenter retrospective study of eribulin in real life. Springerplus. 2016; 5:59. https://doi.org/10.1186/s40064-016-1700-0.

28. Dell'Ova M, De Maio E, Guiu S, Roca L, Dalenc F, Durigova A, Pinguet F, Bekhtari K, Jacot W, Pouderoux S. Tumour biology, metastatic sites and taxanes sensitivity as determinants of eribulin mesylate efficacy in breast cancer: results from the ERIBEX retrospective, international, multicenter study. Bmc Cancer. 2015; 15:659. https://doi. org/10.1186/s12885-015-1673-3.

29. Smith JW 2nd, Vukelja S, Hoffman AD, Jones VE, McIntyre K, Berrak E, Song JX, O'Shaughnessy J. Phase II, Multicenter, Single-Arm, Feasibility Study of Eribulin Combined With Capecitabine for Adjuvant Treatment in Estrogen Receptor-Positive, Early-Stage Breast Cancer. Clin Breast Cancer. 2016; 16:31-7. https://doi. org/10.1016/j.clbc.2015.07.007.

30. Wilks S, Puhalla S, O'Shaughnessy J, Schwartzberg L, Berrak E, Song J, Cox D, Vahdat L. Phase 2, multicenter, single-arm study of eribulin mesylate with trastuzumab as first-line therapy for locally recurrent or metastatic HER2positive breast cancer. Clin Breast Cancer. 2014; 14:40512. https://doi.org/10.1016/j.clbc.2014.04.004.

31. Gitlitz BJ, Tsao-Wei DD, Groshen S, Davies A, Koczywas M, Belani CP, Argiris A, Ramalingam S, Vokes EE, Edelman M, Hoffman P, Ballas MS, Liu SV, et al. A phase II study of halichondrin B analog eribulin mesylate (E7389) in patients with advanced non-small cell lung cancer previously treated with a taxane: a California cancer consortium trial. J Thorac Oncol. 2012; 7:574-8. https://doi. org/10.1097/JTO.0b013e31823f43ca.
32. Dranitsaris G, Beegle N, Kalberer T, Blau S, Cox D, Faria C. A comparison of toxicity and health care resource use between eribulin, capecitabine, gemcitabine, and vinorelbine in patients with metastatic breast cancer treated in a community oncology setting. J Oncol Pharm Pract. 2015; 21:170-7. https://doi.org/10.1177/1078155214525369.

33. Fabi A, Moscetti L, Ciccarese M, Caramanti M, Salesi N, La Verde N, Russillo M, Generali D, Scandurra G, Vari S, Pacetti U, Cognetti F, Giannarelli D. Eribulin in heavily pretreated metastatic breast cancer patients and clinical/ biological feature correlations: impact on the practice. Future Oncol. 2015; 11:431-8. https://doi.org/10.2217/ fon.14.271.

34. Hensley ML, Kravetz S, Jia X, Iasonos A, Tew W, Pereira L, Sabbatini P, Whalen C, Aghajanian CA, Zarwan C, Berlin S. Eribulin mesylate (halichondrin B analog E7389) in platinum-resistant and platinum-sensitive ovarian cancer: a 2-cohort, phase 2 study. Cancer. 2012; 118:2403-10. https:// doi.org/10.1002/cncr.26569.

35. Ates O, Babacan T, Kertmen N, Sarici F, Cenoli A, Akin S, Karakas Y, Kilickap S, Ozisik Y, Sever AR, Aksoy S, Altundag K. Efficacy and safety of eribulin monotherapy in patients with heavily pretreated metastatic breast cancer. J Buon. 2016; 21:375-81.

36. Kawai A, Araki N, Naito Y, Ozaki T, Sugiura H, Yazawa Y, Morioka H, Matsumine A, Saito K, Asami S, Isu K. Phase 2 study of eribulin in patients with previously treated advanced or metastatic soft tissue sarcoma. Jpn J Clin Oncol. 2017; 47:137-44. https://doi.org/10.1093/jjco/ hyw 175.

37. Kaklamani VG, Jeruss JS, Hughes E, Siziopikou K, Timms KM, Gutin A, Abkevich V, Sangale Z, Solimeno C, Brown KL, Jones J, Hartman AR, Meservey C, et al. Phase II neoadjuvant clinical trial of carboplatin and eribulin in women with triple negative early-stage breast cancer (NCT01372579). Breast Cancer Res Treat. 2015; 151:62938. https://doi.org/10.1007/s10549-015-3435-y.

38. Kessler L, Falato C, Margolin S, Bergh J, Foukakis T. A retrospective safety and efficacy analysis of the first patients treated with eribulin for metastatic breast cancer in Stockholm, Sweden. Acta Oncol. 2015; 54:522-9. https:// doi.org/10.3109/0284186x.2014.973063.

39. Lorusso V, Cinieri S, Latorre A, Porcu L, Del Mastro L, Puglisi F, Barni S. Efficacy and safety of eribulin in taxanerefractory patients in the 'real world'. Future Oncol. 2017; 13:971-8. https://doi.org/10.2217/fon-2016-0530.

40. McIntyre K, O’Shaughnessy J, Schwartzberg L, Gluck S, Berrak E, Song JX, Cox D, Vahdat LT. Phase 2 study of eribulin mesylate as first-line therapy for locally recurrent or metastatic human epidermal growth factor receptor 2-negative breast cancer. Breast Cancer Res Treat. 2014; 146:321-8. https://doi.org/10.1007/s10549-014-2923-9.

41. Gamucci T, Michelotti A, Pizzuti L, Mentuccia L, Landucci E, Sperduti I, Di Lauro L, Fabi A, Tonini G, Sini V, Salesi N, Ferrarini I, Vaccaro A, et al. Eribulin mesylate in 
pretreated breast cancer patients: a multicenter retrospective observational study. J Cancer. 2014; 5:320-7. https://doi. org/10.7150/jca.8748.

42. Moscetti L, Mentuccia L, Vici P, Quadrini S, Sperduti I, Pizzuti L, Fabbri MA, Vaccaro A, Maugeri-Sacca M, Barba M, Sergi D, Zampa G, Gamucci T. ESAS and FACT-B in eribulin-treated metastatic breast cancer patients: a multicenter, prospective and observational study. Future Oncol. 2017; 13:1517-1525. https://doi.org/10.2217/fon-2017-0062.

43. Prestifilippo A, Grippaldi D, Blanco G, Memeo L, Puliafito I, Giuffrida D. Eribulin efficacy based on type of metastatic site: a real-life study in heavily pretreated metastatic breast cancer. Future Oncol. 2017; 13:5-10. https://doi. org/10.2217/fon-2017-0017.

44. Quaquarini E, Sottotetti F, D’Ambrosio D, Malovini A, Morganti S, Marinello A, Pavesi L, Frascaroli M. Eribulin across multiple lines of chemotherapy: a retrospective study on quality of life and efficacy in metastatic breast cancer patients. Future Oncol. 2017; 13:11-23. https://doi. org/10.2217/fon-2016-0517.

45. Inoue K, Saito T, Okubo K, Kimizuka K, Yamada H, Sakurai T, Ishizuna K, Hata S, Kai T, Kurosumi M. Phase II clinical study of eribulin monotherapy in Japanese patients with metastatic breast cancer who had well-defined taxane resistance. Breast Cancer Res Treat. 2016; 157:295-305. https://doi.org/10.1007/s10549-016-3808-x.

46. Schoffski P, Ray-Coquard IL, Cioffi A, Bui NB, Bauer S, Hartmann JT, Krarup-Hansen A, Grunwald V, Sciot R, Dumez H, Blay JY, Le Cesne A, Wanders J, et al. Activity of eribulin mesylate in patients with soft-tissue sarcoma: a phase 2 study in four independent histological subtypes. Lancet Oncol. 2011; 12:1045-52. https://doi.org/10.1016/ s1470-2045(11)70230-3.

47. Vahdat LT, Pruitt B, Fabian CJ, Rivera RR, Smith DA, TanChiu E, Wright J, Tan AR, Dacosta NA, Chuang E, Smith J, O'Shaughnessy J, Shuster DE, et al. Phase II study of eribulin mesylate, a halichondrin B analog, in patients with metastatic breast cancer previously treated with an anthracycline and a taxane. J Clin Oncol. 2009; 27:295461. https://doi.org/10.1200/jco.2008.17.7618.

48. Dybdal-Hargreaves NF, Risinger AL, Mooberry SL. Eribulin mesylate: mechanism of action of a unique microtubuletargeting agent. Clin Cancer Res. 2015; 21:2445-52. https:// doi.org/10.1158/1078-0432.ccr-14-3252.

49. Ueda S, Saeki T, Takeuchi H, Shigekawa T, Yamane T, Kuji I, Osaki A. In vivo imaging of eribulin-induced reoxygenation in advanced breast cancer patients: a comparison to bevacizumab. Br J Cancer. 2016; 114:12128. https://doi.org/10.1038/bjc.2016.122.

50. Taylor MA, Lee YH, Schiemann WP. Role of TGFbeta and the tumor microenvironment during mammary tumorigenesis. Gene Expr. 2011; 15:117-32.

51. Crawford J, Caserta C, Roila F. Hematopoietic growth factors: ESMO Clinical Practice Guidelines for the applications. Ann Oncol. 2010; 21:v248-51. https://doi. org/10.1093/annonc/mdq195.

52. Aapro MS, Bohlius J, Cameron DA, Dal Lago L, Donnelly JP, Kearney N, Lyman GH, Pettengell R, TjanHeijnen VC, Walewski J, Weber DC, Zielinski C. 2010 update of EORTC guidelines for the use of granulocytecolony stimulating factor to reduce the incidence of chemotherapy-induced febrile neutropenia in adult patients with lymphoproliferative disorders and solid tumours. Eur J Cancer. 2011; 47:8-32. https://doi.org/10.1016/j. ejca.2010.10.013.

53. Lyman $\mathrm{GH}$, Lyman $\mathrm{CH}$, Agboola O. Risk models for predicting chemotherapy-induced neutropenia. Oncologist. 2005; 10:427-37. https://doi.org/10.1634/ theoncologist.10-6-427.

54. Liberati A, Altman DG, Tetzlaff J, Mulrow C, Gotzsche PC, Ioannidis JP, Clarke M, Devereaux PJ, Kleijnen J, Moher D. The PRISMA statement for reporting systematic reviews and meta-analyses of studies that evaluate health care interventions: explanation and elaboration. Plos Med. 2009; 6:e1000100. https://doi.org/10.1371/journal.pmed.1000100.

55. Stang A. Critical evaluation of the Newcastle-Ottawa scale for the assessment of the quality of nonrandomized studies in meta-analyses. Eur J Epidemiol. 2010; 25:603-5. https:// doi.org/10.1007/s10654-010-9491-z.

56. Higgins JP, Thompson SG, Deeks JJ, Altman DG. Measuring inconsistency in meta-analyses. BMJ. 2003; 327:557-60. https://doi.org/10.1136/bmj.327.7414.557.

57. Egger M, Davey Smith G, Schneider M, Minder C. Bias in meta-analysis detected by a simple, graphical test. Bmj. 1997; 315:629-34.

58. Begg CB, Mazumdar M. Operating characteristics of a rank correlation test for publication bias. Biometrics. 1994; 50:1088-101.

59. Wetterslev J, Thorlund K, Brok J, Gluud C. Trial sequential analysis may establish when firm evidence is reached in cumulative meta-analysis. J Clin Epidemiol. 2008; 61:6475. https://doi.org/10.1016/j.jclinepi.2007.03.013.

60. Brok J, Thorlund K, Wetterslev J, Gluud C. Apparently conclusive meta-analyses may be inconclusive--Trial sequential analysis adjustment of random error risk due to repetitive testing of accumulating data in apparently conclusive neonatal meta-analyses. Int J Epidemiol. 2009; 38:287-98. https://doi.org/10.1093/ije/dyn188.

61. Brok J, Thorlund K, Gluud C, Wetterslev J. Trial sequential analysis reveals insufficient information size and potentially false positive results in many meta-analyses. J Clin Epidemiol. 2008; 61:763-9. https://doi.org/10.1016/j. jclinepi.2007.10.007.

62. Fu W, Zhuo ZJ, Chen YC, Zhu J, Zhao Z, Jia W, Hu JH, Fu K, Zhu SB, He J, Liu GC. NFKB1 -94insertion/deletion ATTG polymorphism and cancer risk: Evidence from 50 case-control studies. Oncotarget. 2017; 8:9806-22. https:// doi.org/10.18632/oncotarget.14190. 